\title{
Integrated Framework to Study Efficient Spectral Estimation Techniques for Assessing Spectral Efficiency Analysis
}

\author{
Kantipudi MVV Prasad \\ Research Scholar \\ Visvesvaraya Technological University \\ Belgaum, India
}

\author{
Dr. H.N. Suresh \\ Prof., Dept. of Instrumentation \\ Bangalore Institute Technology, \\ Bangalore, India
}

\begin{abstract}
The advanced network applications enable software driven spectral analysis of non-stationary signal or processes which precisely involves domain analysis with the purpose of decomposing a complex signal coefficients into simpler forms. However, the proper estimation of power coefficients over frequency components of a random signal leads to provide very useful information required in various fields of study. The complex design constraints associated with conventional parametric models such as Dynamic Average Model, Autoregressive MA, etc. for multidimensional spectral estimation using adaptive filters leads to a situation where higher computational complexities generate significant overhead on the systems. Therefore, the proposed study aims to formulate an efficient framework intended to derive a fast algorithm for processing Adaptive Capon and Phase Estimator (APES). The proposed method is applied to a non-stationary signal which is random. Further, the adaptive estimation of power spectra along with more accurate spectral efficiency has been identified in case of APES. An extensive performance evaluation followed by a comparative analysis has been performed by obtaining the values from different spectral estimation techniques, such as APES, PSC, ASC, and CAPON. Moreover, the framework ensures that unlike others, APES is subjected to attain superior signal quality regarding Power Spectral Density (PSD) and Signal to Noise Ratio (SNR) while achieving very less amount of Mean Square Error (MSE). It also exhibits comparatively low convergence speed and computational complexity as compared to its legacy versions.
\end{abstract}

Keywords-Amplitude and phase estimation; ASC; capon spectral estimator; spectral estimation; PSC

\section{INTRODUCTION}

Spectral analysis of signals is the measurement of power spectral components further analyzed to investigate the frequency coefficients of a random signal. The power distribution over a non-stationary signal eases the computation of frequency components.

However, the large scope of its applicability extended into various fields of study for software-driven electronic devices including Speech Analysis, Medicine, RADAR, and SONAR communications, etc. The prime reason lies in the fact that the frequency content of an observed signal can provide very useful information in the fields like multidimensional intelligence Naval and military communications [1], [2]. A data independent method namely Periodogram was initially developed by the author named Arthur Schuster with the purpose of estimating spectral coefficients of a non-stationary signal efficiently. The numerical computing method which is applied to a synthetic signal has adopted the concept of Fourier transform followed by efficient utilization of FFT algorithm [3]. However, the algorithm is claimed to have a limited scope of applications due to various factors such as poor resolution and high side lobe problems. This situation further leads to a scenario, where retrieval of significant information by analyzing signal coefficients becomes entirely unfeasible.

An in-depth investigational study gives an insight into the fact that the conventional data-dependent (adaptive) methods for both non-parametric and parametric approaches attain superior performance efficiency in comparison with the conventional data independent methods like Periodogram. Adaptive data dependent methodologies are also claimed to achieve optimal computational cost. The applicability of dataadaptive approaches further leads to improve the spectrum quality of a signal significantly and helps to retrieve more information under study. Therefore, it has gained the interest among more researchers to explore its applicability towards mitigating issues of spectral estimation. These advantages have led to increasing interest in data-adaptive approaches towards the problem of spectral estimation. The proposed study thereby formulated a novel framework to access the performance efficiency of the conventional APES technique and determine the quality signal concerning PSD and computational complexity perspectives [4]. The study also gives insight into the in-depth performance analysis of conventional PSC, ASC and Capon estimation methods while improving the SNR as well as reducing the MSE of a nonstationary process. The experimental outcomes precisely exhibit the performance efficiency of the APES method on evaluating spectral correlation (SC) and effective spectral growth regarding SNR and PSD [5]. The paper is organized in a way where Section II discusses the essentials of the spectrum estimation followed by existing survey highlighted in Section III. However, Section IV discusses the conceptual framework for APES spectrum estimation. Finally, Section V discusses the outcomes of the study followed by Section VI that discusses conclusion and future work. 


\section{BACKGROUND}

Most phenomena of signals that occur in nature or practice are typically random and are best modeled as random signals. However, examples of such random signals include various non-deterministic processes, but they are not limited to speech/audio signals and thermal noise generated by electronic devices. Due to the random fluctuation of these signals, they are best characterized regarding statistical averages. The autocorrelation function of a random process is a statistical average used for characterizing these random signals in the time field. A closer look into the estimated PSD spectrum shows that how frequency contents are distributed over periodic time. The spectral estimation thereby involves a process to approximate the uniform distribution of frequency components of an arbitrary signal. The process of estimating the power distribution over frequency components of a nonstationary process thereby exhibits the power band of a signal.

Power spectral estimation has applications in many fields. Speech signals which are periodic are analyzed using the spectrogram. In case of frequency domain analysis provides useful information that can lead to speech recognition and generation. In the sensing fields of RADAR and SONAR, the spectral content of received signals may provide information about the targets of interest in a given scene of interest (see Fig. 1). Also, the power spectrum of signals may provide information about radio frequency interference in such a signal and hence lead to effective suppression of the interference. In MEDICINE field, power band of EEG signals can be used to evaluate the different sleep cycles in humans [6]. These can/are used to investigate and study narcoleptic (a disease characterized by the inability to properly regulate sleep-wake cycles) patients. More recently these are used in audio.

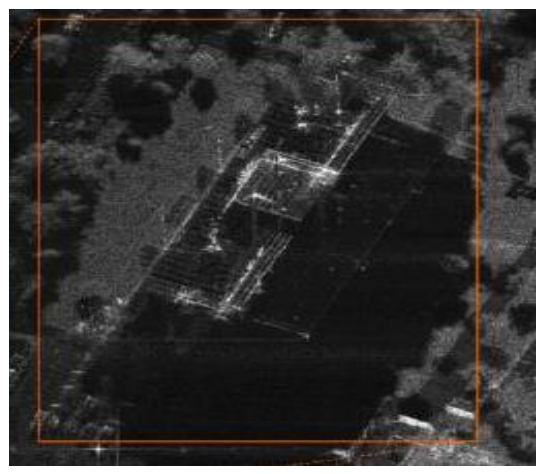

Fig. 1. Synthetic aperture radar imaging.

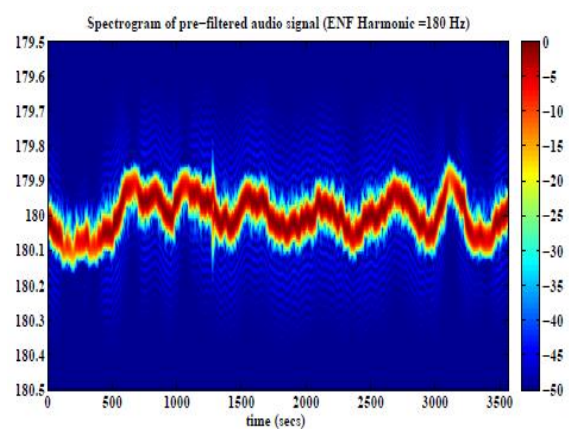

Fig. 2. Spectrogram.
The spectrogram of the audio signal can indicate the presence of the electric network frequency (see Fig. 2), which can be used for digital audio authentication. Synthetic aperture radar imaging: (A) Photograph of the object at 45 degrees (B) SAR image formed using Spectral Estimation (FFT) (Fig. 1.).

There are two broad approaches to manage spectral coefficient estimation. The principal approach is known as the non-parametric technique, and the other is known as the parametric strategy. However, the non-parametric techniques expect no earlier data about the information, while the parametric strategies accept a particular model of the information, which then outcomes in an issue of parameter estimation. The parametric strategies are more exact than the traditional non-parametric systems when the expected model is precise. Notwithstanding, they perform inadequately when there are errors in the information modeling.

\section{A. Problem Formulation}

$$
\text { Let }\{Z n, \vec{n}, n=0,1, \ldots, N-1, \vec{n}=0,1, \ldots, \vec{N}-1\}
$$

Denote a 2-D discrete-time data sequence. For a frequency pair $(\omega, \varpi)_{\text {of interest, we model }} Z n, \vec{n}$ as:

$$
\begin{gathered}
Z n, \vec{n}=\alpha(\omega, \varpi) e j(n w+n w)+w_{n, \bar{n}}(\omega, \varpi), \\
n=0,1, \ldots, N-1, \vec{n}=0,1, \ldots, \vec{N}-1
\end{gathered}
$$

Where, $\alpha(\omega, \varpi)_{\text {denotes the complex amplitude of a 2-D }}$ sinusoid with frequency $(\omega, \varpi)$ and $w n, \vec{n}(\omega, \varpi)_{\text {denotes the }}$ unmodeled noise and interference at the frequency $(\omega, \varpi)$. The problem of interest is to obtain the estimate of $\alpha(\omega, \varpi)_{\text {from }}$ the 2-D data sequence for all $(\omega, \varpi)$ of interest. In 2-D SAR imaging applications, for example, $\alpha(\omega, \varpi)$ would be proportional to the radar cross-section of a target scatterer located at a range proportional to $\omega$ and cross-range proportional to $\varpi$.

\section{B. Energy Spectral Density}

Consider a signal $x[\eta]$ (discrete) with finite energy, that is,

$$
E=\sum_{n=-\infty}^{\infty}|x[\eta]|^{2}<\infty
$$

Then its discrete-time Fourier transforms (DTFT) exists and is given by:

$$
X(\omega)=\sum_{n=-\infty}^{\infty} \mid x[\eta] e^{-j w n}
$$

Where, $\omega$ is the angular frequency variable measured in radians per sample? From Parseval's theorem equation (1-1) can be written as: 


$$
E=\sum_{n=-\infty}^{\infty}|x[\eta]|^{2}=\frac{1}{2 \pi} \int_{-\pi}^{\pi}|X(\omega)|^{2}
$$

From the equation above the energy spectral density of $x[\eta]_{\text {which is the distribution of the energy of the signal of }}$ frequency is therefore defined as:

$$
S_{x x}(\omega)=|X(\omega)|^{2}
$$

Note that the ESD $S_{x x}(\omega)$ can be written as the computation of Fourier transform associative autocorrelation sequence $\Gamma_{x x}(k)$ of the signal

$$
x[\eta] .
$$

Where,

$$
\Gamma_{x x}(k)=\sum_{n=-\infty}^{\infty} x^{*}[\eta] x[n-k]
$$

The analysis above is expressly for signals with finite energy (deterministic signals). However, signals typically encountered in applications are characterized as stochastic processes and do not have finite energy and hence do not possess a Fourier transform. These random signals, however, possess and average power can be described by their PSD.

\section{Power Spectral Density}

Consider a stationary stochastic process $y[\eta]$, where $E\{y[\eta]\}=0$ for all $\eta$. The auto covariance function (same as Auto Correlation function for stationary stochastic process with mean zero) of $y[\eta]$ is given by

$$
\Gamma_{y y}(k)=E\left\{y^{*}[\eta] y[\eta-k]\right\}
$$

Where, $E\{\bullet\}$ is the statistical average over all realizations? The PSD of $y[\eta]$ is well-defined as:

$$
\theta_{y y}(\omega)=\sum_{n=-\infty}^{\infty} \tau_{y y}(k) e^{-j w k}
$$

This simply the Fourier transforms of the AC function. Note that the inverse transform of this PSD gives $\tau_{y y}(k)$ as shown below:

$$
\frac{1}{2 \Pi} \int_{-\pi}^{\pi} \theta_{y y}(\omega) e^{j w k} d w=\sum_{s=-\infty}^{\infty} \tau_{y y}(s)\left[\frac{1}{2 \pi} \int_{-\pi}^{\pi} e^{j w(k-s)} d \omega\right]=\sum_{s=-\infty}^{\infty} \tau_{y y}(s) \delta_{k s}=\tau_{y y}(k)
$$

Where, $\delta$ denotes the Kronecker delta function. Note that the average power of the stochastic process $y[\eta]$ is given by the zero lag o the AC function $\tau_{y y}(0)$ :

$$
E\left\{\left.y[\eta]\right|^{2}\right\}=\tau_{y y}(0)=\frac{1}{2 \pi} \int_{-\pi}^{\pi} \phi_{y y}(\omega) d \omega
$$

This equation (1-9) leads to the motivation for defining the PSD in (1-8). The PSD can also be defined as:

$$
\phi_{y y}(\omega)=\lim _{N \rightarrow \infty} E\left\{\frac{1}{N}\left|\sum_{\eta=1}^{N} y[\eta] e^{-j w n}\right|^{2}\right\}
$$

Which is equivalent to the definition in (1-8) under the assumption that the autocovariance sequence (ACS) $\tau_{y y}(k)$ decays quickly.

\section{EXISTING SURVEY}

Guo et al. [7] investigated the outcome of frequency shifting in an acoustic feedback control system. This work recommends a solution to achieve an unbiased approximation by eliminating the slowly time variable part in adaptive filter estimation.

Kim et al. [8] suggested a less computational improved time-based and spectral constraints approximation methods for SIFT output of radar signals. This method enhanced the spectral resolution of a received radar signal by utilizing the STFT outputs. Similarly, it will also improve the time-based resolution of the complete system for obtained radar signals. The performance of the executed approaches are hypothetically examined, and it accomplishes the low computational complexity is obtained.

Rosado et al. [9] analyzed the presentation of an adaptive multilayer technique for decreasing the coherent noise associated with spectral of ultrasound echoes. The proposed strategy effectively lessened predisposition and coherent noise related to standards for different methodologies, demonstrating its favorable position for spectral investigation of muddled back-scattered signals.

Fu et al. [10] introduced a novel technique for adaptively investigating the time-varying AC of non-stationary signals. This article also gives the information about its applications to time-frequency spectrum analysis. This method implements a local valuation with sliding window method to have a certain bandwidth to investigate time-varying AC locally.

Zhang et al. [11] proposed a novel adaptive Kalman filter based recursive technique to estimate the spectrum for measuring time-varying non-stationary signals. The measurements in Kalman filter are decided adaptively as indicated by the state divisions. The simulation results show that the proposed method achieves a better time-frequency resolution that compared to the traditional spectrum estimations. 
Cheng et al. [12] presented an alteration of traditional least mean squared error (MMSE) inappropriate subtraction calculation by acquainting a versatile averaging variable with precisely evaluate the from the earlier SNR. Execution of the adjusted process concerning the earlier SNR is assessed by contrasting and ordinary phantom subtraction calculation. Enhanced results are gotten as far as discourse quality measures for different sorts of commotion when the timerecurrence fluctuating averaging element, proposed in this paper; the customary subtraction rules are also exhibited.

Bracale et al. [13] concentrated on otherworldly parts of force static converters utilized for both train drives, and assistant administrations can be exceedingly time-changing with ensuing troubles in their location. In this paper, a versatile asymmetric strategy is proposed to ascertain time-changing phantom segments with great precision and adequate computational endeavors. The strategy depends on a versatile system which minimizes the mean square relative blunder of sign estimation. The utilization of the strategy to an assistant static converter demonstrates its high precision and satisfactory computational endeavors.

Alty et al. [14] displayed a computationally effective sliding window time redesigning of the Capon and sufficiency, and stage estimation (APES) coordinated channel bank otherworldly estimators in light of the time-variation relocation structure of the information covariance network. The exhibited calculation shapes a characteristic expansion of the most computationally effective calculation to date and offers a critical computational addition when contrasted with the computational unpredictability connected with the bunch reassessment of the appraisals for every time-upgrade. Moreover, through recreations, the calculation is observed to be numerically better than the time-overhauled precise gauge framed from straightforwardly redesigning the information covariance grid.

The study of Huillery et al. [15] concentrated on an instance of a spectrogram built from a limited length discretetime boisterous sign is introduced. This study expands past takes a shot at negligible insights on two viewpoints: to start with, the most extreme probability appraisal of the commotion is detailed by clear investigation of the likelihood conveyance of the time-recurrence coefficients. Second, the decision of an ideal insignificant subset is examined. The sign versus commotion segregation property of the ghastly kurtosis is utilized to choose a negligible subset which guarantees a reasonable exchange off between the predisposition and the difference of the estimator. The subsequent exhibitions are talked about and contrasted and those of different strategies through numerical recreations on engineered signals. The utilization of the MiniSMaL estimator in a period recurrence recognition method is at last shown on a certifiable sign.

The study of Zhang et al. [16] proposed another versatile Kalman channel based recursive range estimator for measuring a time-differing range of non-stationary signs. The nonstationary sign is demonstrated as a period shifting autoregressive process, and the time-differing parameters are portrayed by smoothness prior's model. Another Kalman channel calculation with a variable number of estimations is utilized to recursively figure out the TVAR coefficients and after that, the time-differing range is formulated. The quantity of estimations in the Kalman channel is resolved adaptively as per the state gauge subsidiaries. Moreover, a quick QR disintegration calculation is created to decrease the math unpredictability of the proposed KFVNM calculation. Reenactment results demonstrate the proposed Kalman channel based recursive range estimator can accomplish a superior time-recurrence determination than the customary parametric range estimations. Its potential application to power quality checking is likewise shown.

Glentis et al. [17] proposed quick calculations for versatile Capon and adequacy and stage estimation (APES) techniques for efficient examination of time fluctuating signs. A quick, steady, and non-recursive formulae are inferred considering of time moving properties of the related variables. As a result, productive recurrence space recursive slightest squares (RLS) based, and also quick RLS based calculations for the versatile estimation of the force spectra are created. Soundness issues of the recurrence area estimators are considered, and adjustment techniques are proposed. The computational intricacy of the proposed calculations is lower than applicable existing strategies. The execution of the proposed calculations is exhibited through broad reenactments.

The study of Resende et al. [18] introduced a structure that offers ascend to unearthly gauges that speak to the genuine basic range with preferred devotion over routine LS strategies by permitting a subjective exchange off between differences of phantom gauges and following capacity of the estimator along with the recurrence area. The straight forecast mistake is deteriorated through a force correlative channel bank, and segments of every band are broke down by various window lengths, permitting long windows to track gradually differing signs and short windows to watch firstly shifting parts. The connection network of the info sign is appeared to fulfill both time-upgrade and request overhaul properties for rectangular windowing capacities, and an RLS calculation given every property is displayed. Additionally, reenactments looking at the execution of customary and the proposed multi-band LS strategies are delineated and examined.

\section{CONCEPTUAL FRAMEWORK}

The proposed study aims to formulate a Numerical computing framework for estimating spectrum components of a synthetic signal using Capon, PSC, APES and ASC techniques. The study intended to perform spectral estimations by reducing the (MSE) of APES linear regression models. The proposed system aims to design a novel framework is integrated with different significant spectral estimation frameworks, e.g., Capon, PSC, APES, and ASC to perform spectral estimation on non-stationary signals by reducing the (MSE) of all linear regression models. It also performs a comparative analysis in between the conventional state of art spectral estimation processes to compute bit error rate (BER) concerning SNR. Moreover, the proposed model also applies a linear shrinkage based approach (includes both Capon and APES Spectral Estimators) to intensify the spectral quality of an input signal. This section of the study discusses the conceptual model intended to carry out spectral estimation of an input sinusoidal 
signal by applying four different spectral estimation methodologies such as PSC, ASC, CAPON, and APES. It also highlights a comparative analysis to ensure the effectiveness of the proposed integrated framework for APES estimation concerning reducing MSE and enhancing the signal quality.

To understand the error pattern with the performance metric (MSE), the filter length (FL) and signal to SNR measurement are required for the given signal input.

Spectral analysis associated with signal components especially for random signals provides necessary information about the frequency and power components (PSD). The obtained values can be further utilized for numerical integration, analytical modeling, prediction and filtering of the deserved random signal. The prime objective of the spectral estimation process is to formulate a correlation between the spectral components to estimate the spectral density of a random signal concerning different time sample sequence. It deals with the computation of frequency components to determine the significant spectrum component of a random signal.

The following Fig. 3 depicts a pictorial representation of the proposed system shows how the experimental process has been carried out by evaluating the different set of operations during the computation process. It includes generation of the sinusoidal signal, which is further interpreted for the computation of PSD. The model performs Monte-Carlo simulation to perform numerical integration on PSC, CAPON, APES, and ASC. Finally, the proposed system SC Density of the input signals by applying the four algorithms and compute the MSE. SNR, MSE and filter length (M) are considered as the performance parameters, respectively. The MSE value has been computed using a function, which considers the filter length as input parameters.

Fig. 4 represents a flow diagram of the proposed model where a different set of operations such as SAR processing, spectral equalization, and signal chipping are performed. Signal chipping is used to segregate a set frequency components for better resolution of computation. The framework also considers 2-D DFT and re-sampling for construction of signal with better resolution.

After performing 2D DFT different spectral estimation techniques are evaluated to generate the consecutive spectrum (i.e., PSD and PSC). A component called as signal mosaicking is applied on the retrieved signal for re-shaping. The following is the algorithm designed and implemented in a numerical computing framework to evaluate the performance efficiency of the APES method.

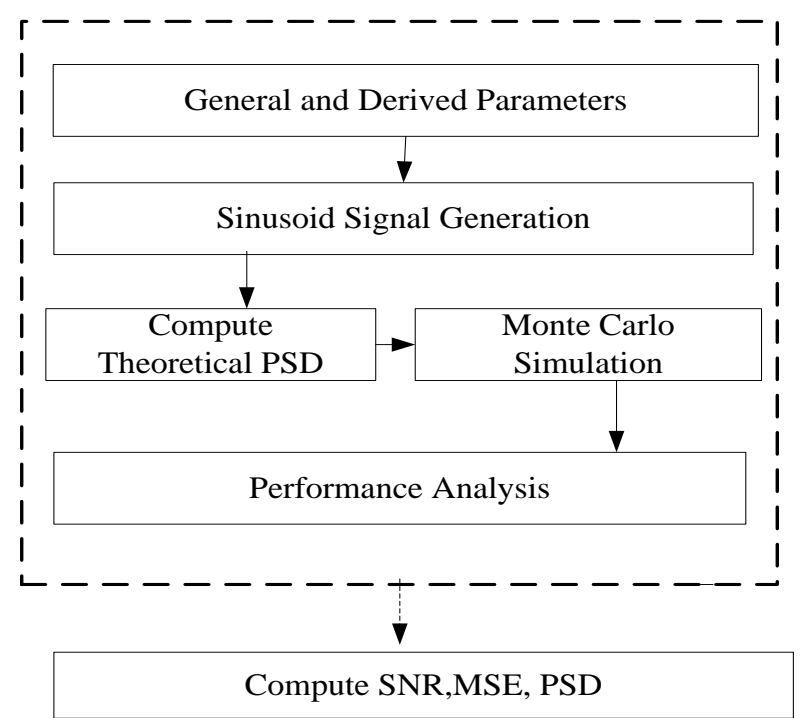

Fig. 3. System architecture of the proposed method.

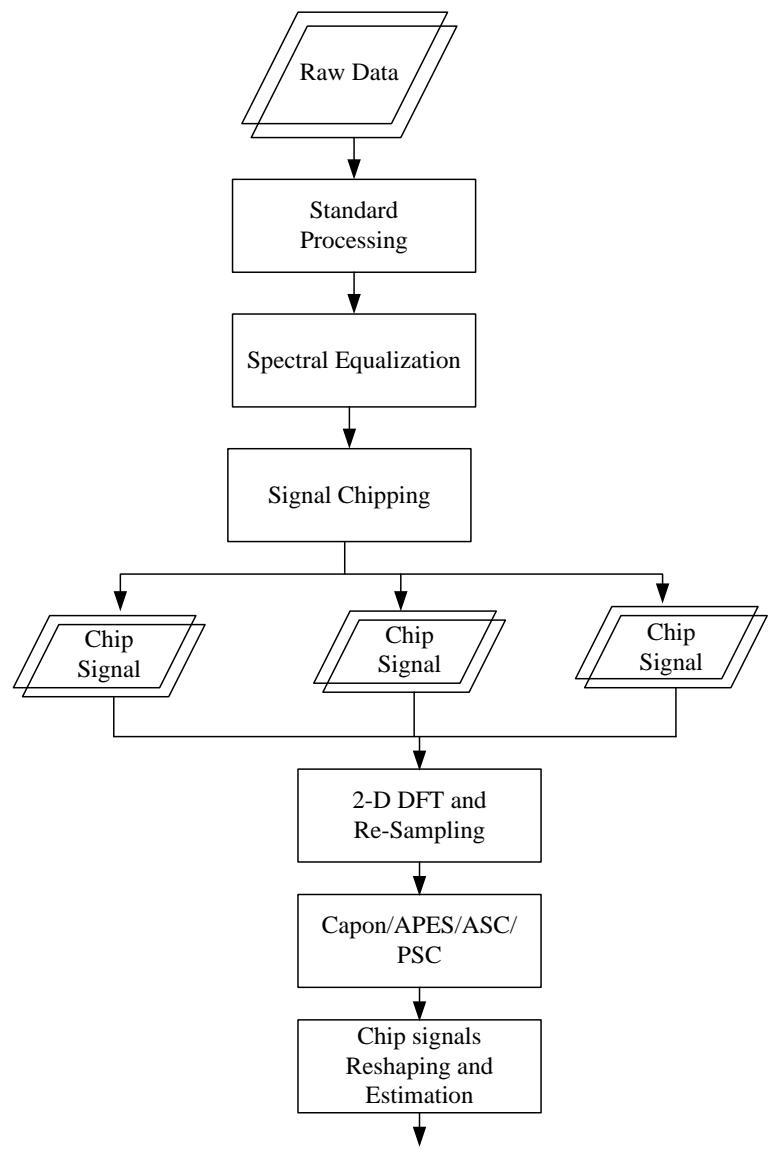

Fig. 4. Flowchart of the proposed method. 


\section{Mathematical Algorithm Design: An Adaptive Framework for Spectral Estimation}

Input: N (Number of Samples), $\mathrm{f}_{\mathrm{s}}$ (Sampling Frequency), M (Filter Length), A (SNR)

Output: Performance Evaluation of Spectral Estimation Techniques on non-stationary signals. (Capon, ASC, APES, PSC)

\section{PROCEDURE:}

\section{START}

1. Input Number of samples $\leftarrow \mathrm{N}$

2. Input sampling frequency $\leftarrow \mathrm{f}_{\mathrm{s}}$

3. Input Sample SNR $\leftarrow$ snr

4. Input Filter Length $\leftarrow \mathrm{M}$

5. Generated signal SNR, matrix $A(1 \times 2) \leftarrow[1,1]$

6. Generated signal Frequency, matrix $\mathrm{f}(1 \times 2) \leftarrow[0.3,0.2]$

7. trails $=10$

8. Sampling period, $\mathrm{Ts} \leftarrow 1 / \mathrm{fs}$

9. $\mathrm{nT}(1 \times \mathrm{N}) \leftarrow \mathrm{Ts}$

10. Generated signal $Y \leftarrow A * \sin \left(2 *\right.$ pi $\left.^{*} \mathrm{f}^{*} \mathrm{nT}\right)$

11. $\mathrm{h} \leftarrow \mathrm{PSD}(\mathrm{Y})$

12. Initiate Monte-Carlo Simulation

13. FOR trails (1: trails)

a. Signal $\mathrm{xin} \leftarrow \mathrm{Y}+$ Gaussian Noise

b. Function SCD

c. Pass In: xin,M,METHOD,fs,res

d. Pass Out: SCD F A

e. Function PSC

f. Pass In: Y, M

g. Pass Out: cross PSD, wave no (k)

h. Function ASC

i. Pass In: Y, M

j. Pass Out: cross PSD, wave no (k)

k. Function CAPON

1. Pass In: Y, M

m. Pass Out: cross PSD, wave no (k)

n. Function APES

o. Pass In: Y, M

p. Pass Out: cross PSD, wave no (k)

14. END ( trails)

15. Plot power spectral distribution, SCD

16. calculate MSE

17. performance parameters.

END

The above algorithm depicts the procedures to compute the performance estimation of different spectral estimation techniques. This method gives the more accurate value of spectral estimates but, the lower resolution than the schemes they based on. A comparative analysis has been performed in between the entire standard APES algorithm and the conventional methods which show that integrated APES outperforms the conventional shrinkage based techniques regarding detection of every frequency and also the amplitude of spectral components. Fig. 3 shows the system design architecture of the proposed method. It contains as an input like general and derived parameters like N, M, A, SNR, f. fs, t, ts, etc. The sinusoidal signal is generated after collecting all the inputs to the system. Then, the next step is to calculate the PSD of the given input signal. Apply Monte Carlo method to simulate the signal. Then add the Gaussian noise to the input signal. Perform the PSC, CAPON, APES and PSC techniques to simulate the experiments. Finally, calculate the SC for the above-mentioned techniques. Estimate the MSE and filter length of the signal. Analyze the given signal using performance parameters like MSE, SNR, and filter length. Fig. 4 shows the flowchart of the proposed method. It gives an explicit conceptual aspect of the proposed framework.

\section{RESULTS AND DISCUSSION}

The computational efficiency offered by the proposed method is illustrated in Fig. 4, where the process involves 
effective SC of APES method is illustrated as well as the spectral estimation of the above algorithm is shown as a function of the sliding window data size $\mathrm{N}$. here the number of observed samples $\mathrm{N}=100$, filter length $\mathrm{M}=8$, sampling frequency $\mathrm{fs}=1, \mathrm{SNR}=35$ is assumed for the implementation purposes. Fig. 5 to 19 shows the result obtained after simulating this proposed framework on a numerical computing tool. It shows that it gives a better spectral estimation for a given non-stationary signals.

Fig. 18 and 19 depicts that APES significantly achieves better outcomes concerning MSE and SNR thus exhibits the conventional spectral estimation techniques.

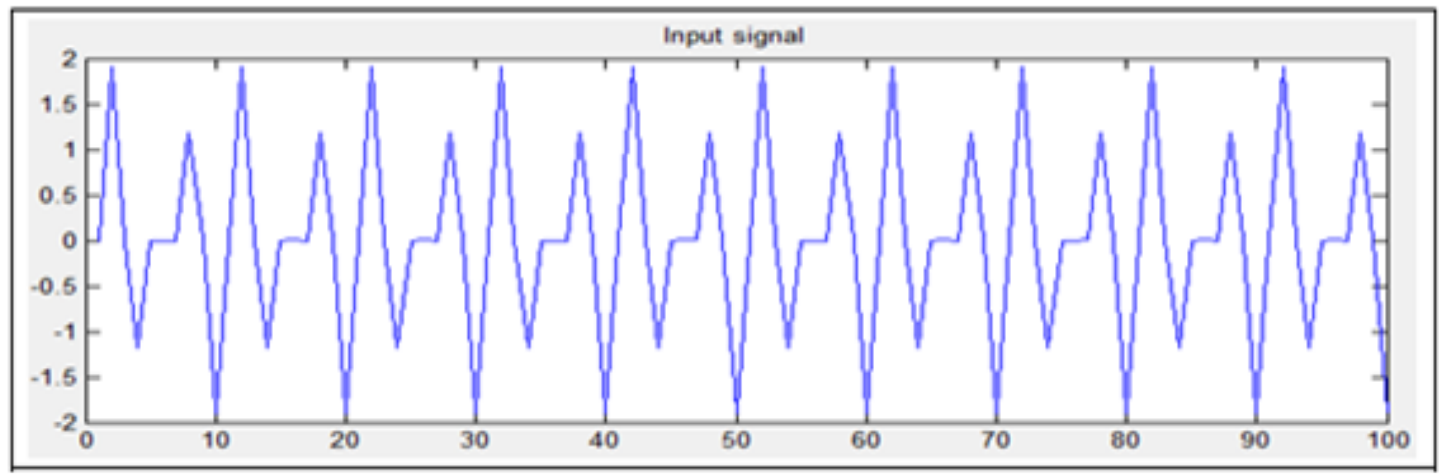

Fig. 5. Input signal.

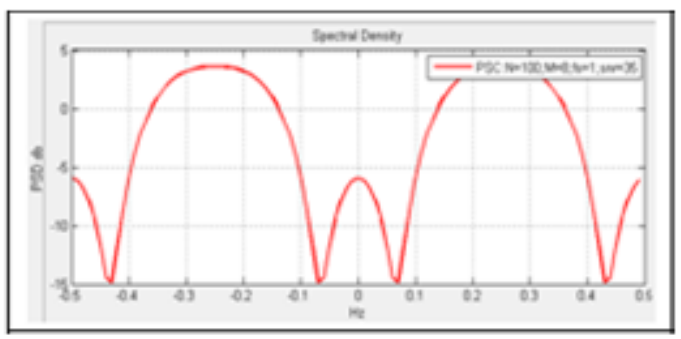

Fig. 6. PSC spectrum estimation.

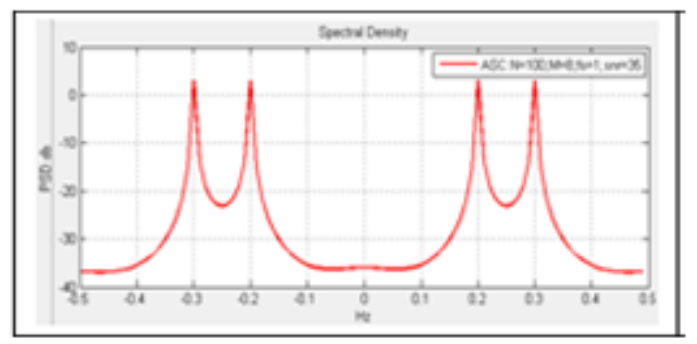

Fig. 7. ASC spectrum estimation.

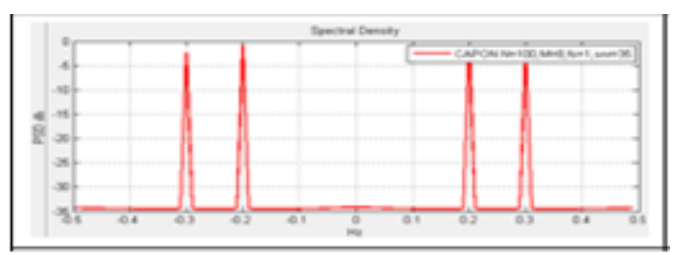

Fig. 8. CAPON spectrum estimation.

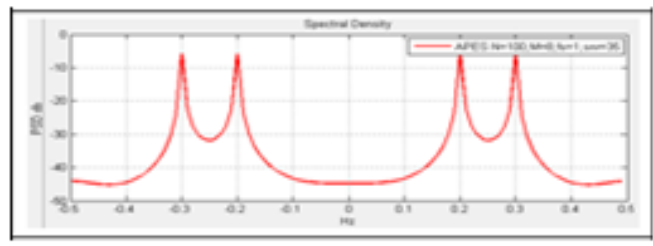

Fig. 9. APES spectrum estimation.

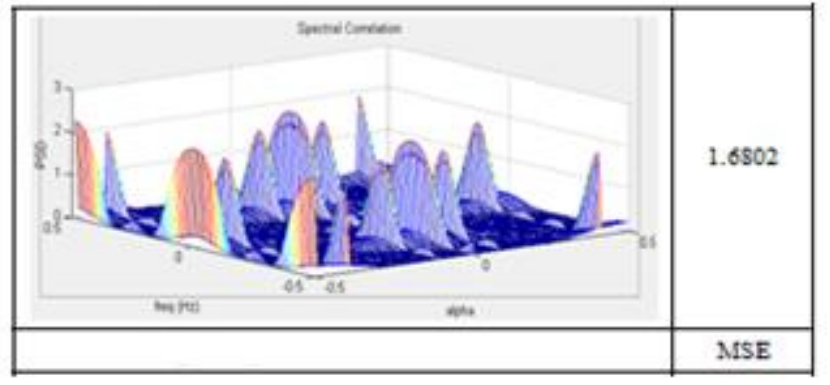

Fig. 10. Spectral correlation for PSC.

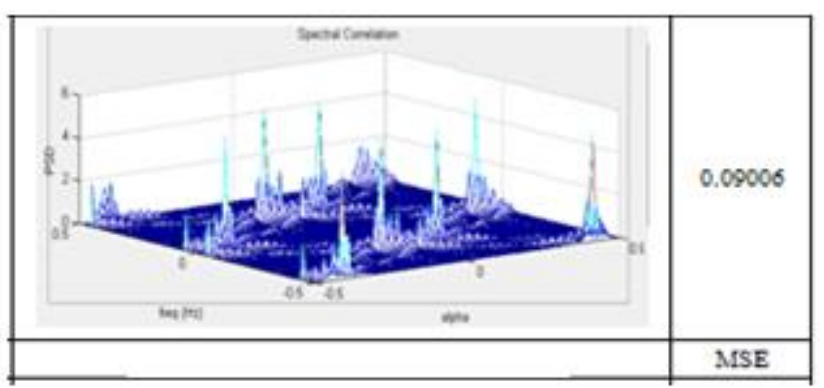

Fig. 11. Spectral correlation for ASC.

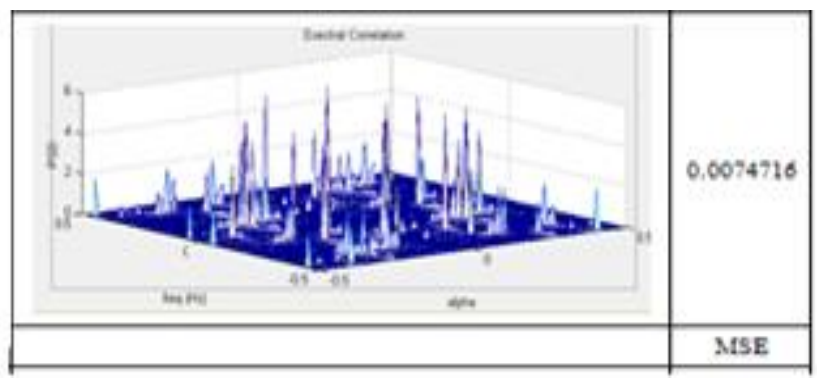

Fig. 12. Spectral correlation for CAPON. 


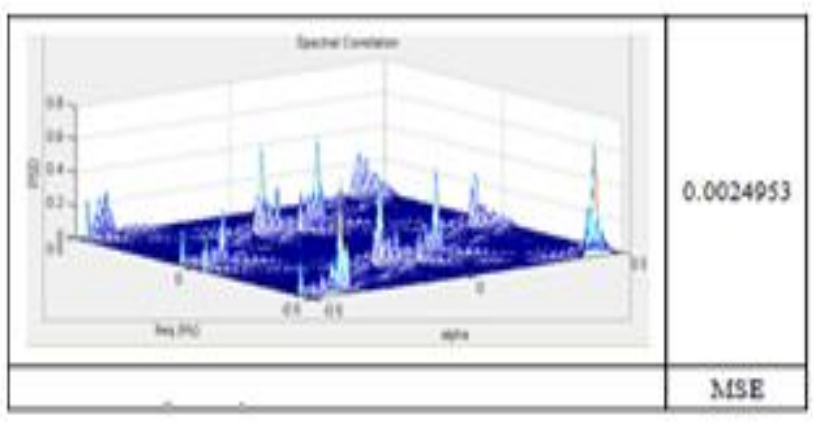

Fig. 13. Spectral correlation for APES.

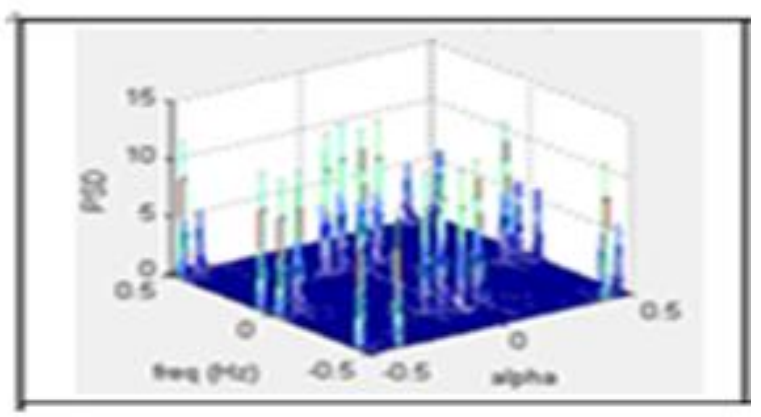

Fig. 14. Spectrum correlation (PSC).

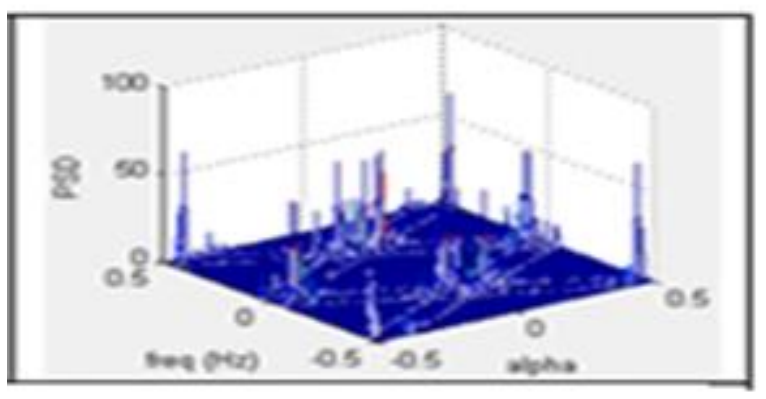

Fig. 15. Spectrum correlation (ASC).

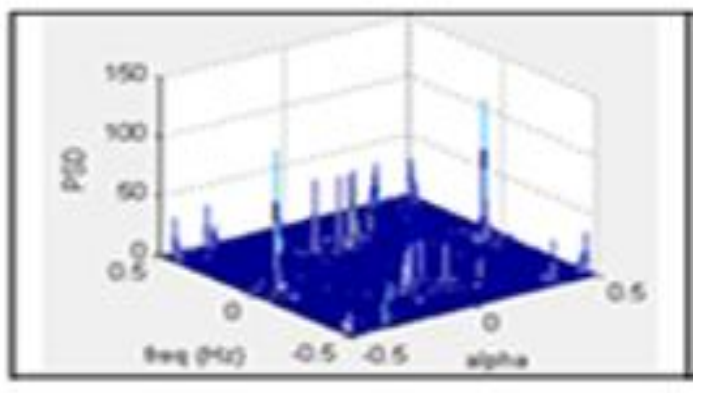

Fig. 16. Spectrum correlation (CAPON).

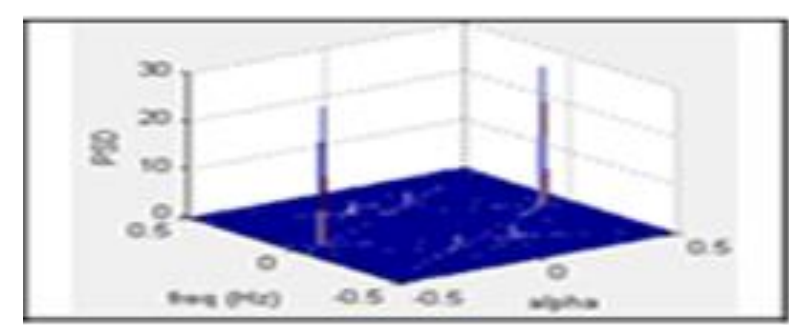

Fig. 17. Spectrum correlation (APES).

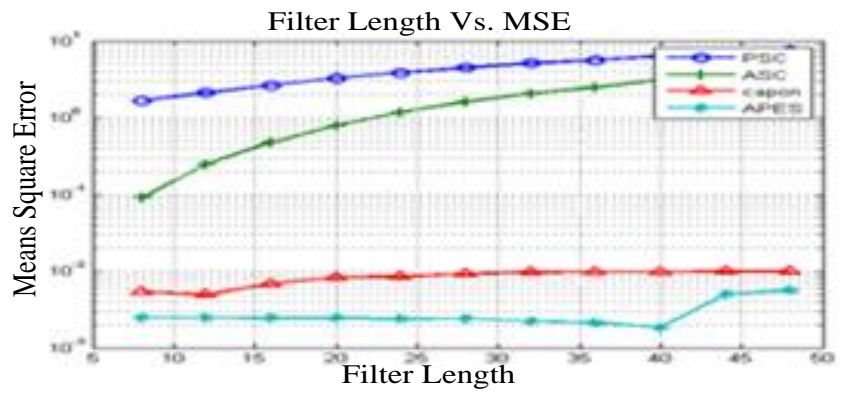

Fig. 18. Filter Length vs. MSE comparison for PSC, ASC, CAPON and APES.

SNR Vs. Mean Square Error

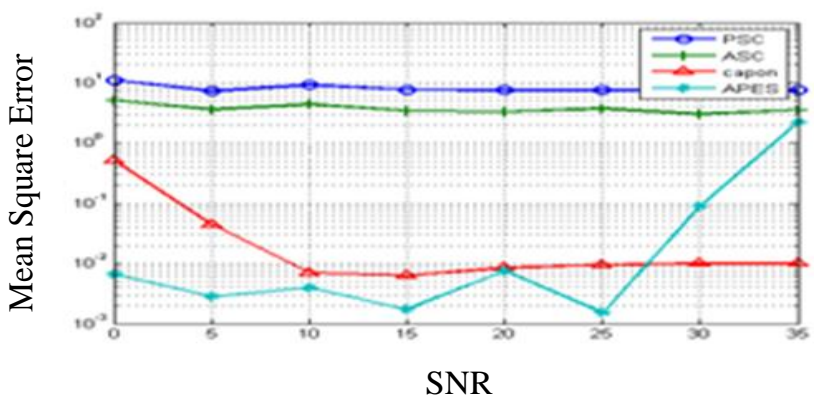

Fig. 19. SNR vs. MSE comparison for PSC, ASC, CAPON and APES.

\section{CONCLUSION AND FUTURE WORK}

The present paper has studied about the effectiveness of existing compressive sensing algorithms that has been seen too.

Further, the study introduced the shrinkage-based APES spectral estimators, termed integrated linear regression framework for APES, by minimizing the MSE of APES in a linear regression shrinkage approach.

Regarding resolution, the proposed framework for APES is found to be a superior method. It has the appealing property that this resolution, even for fairly high target densities, improves as the SNR increases. For applications in which target discrimination is critical, it may be the preferred choice. In particular, in a polarimetric case, APES may be able to discriminate close targets with different polar metric signature where Capon or a traditional matched filter approach cannot. The asymptotic analysis of the proposed APES shows that it 
significantly reduces the computational overhead and obtains efficient convergence speed like RLS. Therefore a closer look at the above Fig. 18 and 19 exhibits that to a large extent the proposed framework for APES achieves very less MSE as compared to the conventional spectral estimation techniques. Future work will focus on testing and apply integrated APES to In SAR, DInSAR, and PolDInSAR. Using large data sets in DInSAR should allow a real-life evaluation of the phase quality of both algorithms. Also, DInSAR in urban areas, where side lobe mitigation is a real issue, and large sets of SLCs are available, can be expected to benefit directly from these algorithms. Another possible research direction is to find criteria and algorithms to automatically select some of the processing parameters.

\section{REFERENCE}

[1] H. Tang and S. Watson, "Cognitive radio networks for tactical wireless communications," Defense Research and Development Canada, 2014.

[2] J.T. Ackerman and K. M-Norris, "Future Trends and US National Security," Air Univ Maxwell AFB Al Air Force Research INST, 2015.

[3] S. Guerrier, J. Skaloud, Y. Stebler, and M-P. V-Feser, "Waveletvariance-based estimation for composite stochastic processes," Journal of the American Statistical Association, Vol. 108, No. 503, pp. 10211030, 2013.

[4] Fevralev, V. Dmitriy, N.N. Ponomarenko, V.V. Lukin, S.K. Abramov, K.O. Egiazarian, and J.T. Astola, "Efficiency analysis of color image filtering," EURASIP Journal on advances in signal processing, No. 1, pp. 41, 2011.

[5] U. Bottigli, R. Chiarucci, B. Golosio, G. L. Masala, P. Oliva, S. Stumbo, D. Cascio, "Superior Performances of the Neural Network on the Masses Lesions Classification through Morphological Lesion Differences," Statistics, Vol. 141, pp.6430, 2006.

[6] R. Xiao and L.Ding, "Evaluation of EEG features in decoding individual finger movements from one hand," Computational and mathematical methods in medicine, 2013.

[7] M. Guo and B. Kuenzle, "On the periodically time-varying bias in adaptive feedback cancellation systems with frequency shifting," IEEE International Conference on Acoustics, Speech and Signal Processing (ICASSP), Shanghai, pp. 539-543, 2016.

[8] B. Kim, S. H. Kong, and S. Kim, "Low Computational Enhancement of STFT-Based Parameter Estimation," In IEEE Journal of Selected Topics in Signal Processing, Vol. 9, No. 8, pp. 1610-1619, 2015.
[9] I. M. Rosado-Mendez, T. J. Hall, and J. A. Zagzebski, "Performance of an adaptive multitaper method for reducing coherent noise in spectral analysis of ultrasound backscattered echoes," IEEE International Ultrasonics Symposium (IUS), Prague, pp. 429-432, 2013.

[10] Z. Fu, Z. Zhang and S. C. Chan, "Estimation of time-varying autocorrelation and its application to time-frequency analysis of nonstationary signals," IEEE International Symposium on Circuits and Systems (ISCAS2013), Beijing, 1524-1527, 2013.

[11] Z. G. Zhang, S. C. Chan and X. Chen, "A new Kalman filter-based recursive method for measuring and tracking time-varying spectrum of no stationary signals," Information, Communications and Signal Processing (ICICS) 9th International Conference on, Tainan, pp. 1-4, 2013.

[12] N. Cheng, W. J. Liu and B. Xu, "An Improved A Priori MMSE Spectral Subtraction Method for Speech Enhancement," 3rd International Workshop on Signal Design and Its Applications in Communications, Chengdu, pp. 373-377, 2007.

[13] A. Bracale, D. Proto, and P. Varilone, "Adaptive Prony Method for Spectrum Estimation of Non-stationary Signals in Traction Systems," EUROCON- The International Conference on "Computer as a Tool, Belgrade, pp. 1550-1553, 2005.

[14] S. R. Alty, A. Jakobsson, and E. G. Larsson, "Efficient time-recursive implementation of matched filterbank spectral estimators," In IEEE Transactions on Circuits and Systems I: Regular Papers, Vol. 52, No. 3, pp. 516-521, 2005.

[15] J. Huillery, F. Millioz, and N. Martin, "Gaussian Noise Time-Varying Power Spectrum Estimation With Minimal Statistics," In IEEE Transactions on Signal Processing, Vol. 62, No. 22, pp. 5892-5906, 2014.

[16] Z. G. Zhang, S. C. Chan and X. Chen, "A new Kalman filter-based recursive method for measuring and tracking time-varying spectrum of nonstationary signals," Information, Communications and Signal Processing (ICICS) 9th International Conference on, Tainan, pp. 1-4, 2013.

[17] G. O. Glentis, "Efficient Algorithms for Adaptive Capon and APES Spectral Estimation," In IEEE Transactions on Signal Processing, Vol. 58, No. 1, pp. 84-96, 2010.

[18] F. G. V. Resende, K. Tokuda, M. Kaneko and A. Nishihara, "RLS algorithms for adaptive AR spectrum analysis based on multi-band decomposition of the linear prediction error," TENCON '96. Proceedings, IEEE Tencon, Digital Signal Processing Applications, Perth, WA, Vol.2, pp. 541-546, 1996. 